\title{
EXISTENCE THEOREMS FOR SEGAL QUANTIZATION VIA SPECTRAL THEORY IN KREIN SPACE
}

\author{
P. BROADBRIDGE'
}

(Received 8 March 1982; revised 16 July 1982)

\begin{abstract}
Segal's unitarizing complex structure $J$ is shown, in the Fermi-Dirac case, to be the orthogonal component in the polar decomposition of the real skew adjoint generator of classical dynamics. It is proven that in the Bose-Einstein case, the classical symplectic dynamics cannot be unitarized unless the generator is similar to a real skew adjoint operator.

With the classical Hamiltonian strictly positive, $J$ is the pseudo-orthogonal component in the polar decomposition of the generator, using spectral theory in Krein space with indefinite metric. Thus, $J$ can be expressed simply in terms of the projection $E(0)$ onto the subspace of classical solutions with negative frequency. This complements the physicists' experience that conceptual difficulties arise when dynamically invariant separation of positive and negative frequency solutions is impossible.
\end{abstract}

\section{Introduction}

In accordance with Hamiltonian mechanics, a classical linear dynamical system consists of a real space $\mathfrak{V}$ with symplectic form $B$, on which there is given a one-parameter group $C(t)$ of symplectic transformations. Following Segal [20-22], quantization according to Bose-Einstein statistics involves the construction of a Weyl system

$$
\checkmark \ni \zeta \rightarrow W(\zeta)
$$

with $W(\zeta)$ unitary operators on a complex Hilbert space $(\mathscr{F},\langle\cdot, \cdot\rangle)$ satisfying the canonical commutation relations in Weyl form

$$
W(\zeta) W(\xi)=\exp \left[\frac{1}{2} i B(\zeta, \xi)\right] W(\zeta+\xi),
$$

\footnotetext{
'School of Physics and Geosciences, W.A.I.T., Hayman Road, South Bentley, W.A. 6102.

(c) Copyright Australian Mathematical Society 1983
} 
and weak ray continuity of $\{W(t \zeta) ; t \in R\}$ for fixed $\zeta$. The Fermion analogue of the above involves the construction of a Hilbert space representation of the canonical anti-commutation relations in Shale-Stinespring form [24]:

$$
[R(\zeta), R(\xi)]_{+}=S(\zeta, \xi) I
$$

with $\zeta$ and $\xi$ arbitrary elements of a real Hilbert space $(\mathcal{V}, S)$.

In the second quantization procedure, $B(\zeta, \xi)$ (or $S(\zeta, \xi)$ ) appears as a scalar multiple of $\operatorname{Im}\langle\zeta, \xi\rangle_{1}\left(\mathrm{Or} \operatorname{Re}\langle\zeta, \xi\rangle_{1}\right)$, with $\zeta$ and $\xi \xi$ velongining to singie particie space, a complex Hilbert space $\left(\mathcal{K},\langle\cdot, \cdot\rangle_{1}\right)$ and $C(t)$ is unitary. Although for infinite classical degrees of freedom, there is a continuum of inequivalent representations [12] of (1.2) or (1.3), there is a natural way of defining a unique abstract $C^{*}$ algebra $\mathbb{Q}$ of second quantized operators. In the Boson case, $\mathbb{Q}$ is the Weyl $C^{*}$ algebra of conceptual observables which was defined by Segal [20-22] while in the Fermion case it is the Clifford $C^{*}$ algebra over $\mathcal{H}$ which was defined by Shale and Stinespring [24].

Given that a complex Hilbert space structure on the classical space $\widetilde{V}$ allows algebraic second quantization to run so naturally, the next question is how to select a physically appropriate Hilbert space representation of $\mathbb{Q}$ in a direct abstract manner. It is now well known [21] that uniqueness of a linear functional $E$ on $Q$ can be guaranteed by the following conditions:

that the generating function $E(W(\zeta))$ is continuous;

that $E$ is time-invariant; $E(W(\zeta(t)))=E(W(\zeta(0)))$;

that in the Gelfand-Naimark-Segal representation $\pi_{E}$ of $Q$ induced by $E$, there exists a continuous one-parameter unitary group $U(t)$ with positive generator and implementing time evolution in $\mathcal{Q}$,

$$
\pi_{E} \circ W(\zeta(t))=U(t)\left[\pi_{E} \circ W(\zeta(0))\right] U^{-1}(t) .
$$

In the Gelfand-Naimark-Segal representation $\pi_{E}$ given by the unique functional $E$ satisfying (1.4), there exists a cyclic vector $\psi_{0} \in \mathscr{F}$ such that $E(A)=$ $\left\langle\psi_{0}, \pi_{E}(A) \psi_{0}\right\rangle$. Then $\psi_{0}$ may be identified with the vacuum in Fock space $\mathscr{F}(\mathcal{H})$ over $\mathcal{H}$, which has a consistent physical interpretation. In the standard Cook construction [7] of Fock space $\mathscr{F}(\mathcal{K})$, the annihilation operators $a(\zeta)$ and their adjoints, the creation operators $a^{\dagger}(\zeta)$ satisfy

$$
\begin{aligned}
& {\left[a(\zeta), a^{\dagger}(\zeta)\right]_{ \pm}=\langle\zeta, \xi\rangle_{1} I,} \\
& {[a(\zeta), a(\xi)]_{ \pm}=\left[a^{\dagger}(\zeta), a^{\dagger}(\xi)\right]=0,}
\end{aligned}
$$

where + and - denote commutation or anti-commutation, appropriate to Bose statistics or to Fermi-Dirac statistics respectively. Then the Segal field operators $\Phi(\zeta)=2^{-1 / 2}\left[a(\zeta)+a^{\dagger}(\xi)\right]$ are essentially self adjoint [19] and satisfy 
$[\Phi(\zeta), \Phi(\xi)]_{-}=i \operatorname{Im}\langle\zeta, \xi\rangle_{1}$ (on a dense domain in $\mathscr{F}$, in the Boson case). For $\alpha \in R$,

$$
\exp [i \alpha \Phi(\zeta)] \exp [i \alpha \Phi(\xi)]=\exp \left[-\frac{1}{2} i \alpha^{2} \operatorname{Im}\langle\zeta, \xi\rangle_{1}\right] \exp [i \alpha \Phi(\zeta)+i \alpha \Phi(\xi)]
$$

$$
[\Phi(\alpha \zeta), \Phi(\alpha \xi)]_{+}=\alpha^{2} \operatorname{Re}\langle\zeta, \xi\rangle_{1} \quad \text { (Fermi-Dirac case) }
$$

Thus, $W(\zeta) \supset \exp [\alpha \Phi(\zeta)]$ provides a Weyl system, conforming with (1.2), provided

$$
\operatorname{lm}\langle\zeta, \xi\rangle_{1}=-\alpha^{-2} B(\zeta, \xi) .
$$

Further, $R(\zeta)=\Phi(\alpha \zeta)$ conforms with (1.3), provided

$$
\operatorname{Re}\langle\zeta, \xi\rangle_{1}=\alpha^{-2} S(\zeta, \xi) .
$$

Since the above procedure of rigorous second quantization runs so smoothly, we are motivated to ask which classical systems ( $\mathfrak{V}, B$ (or $S), C(t))$ can be viewed as complex Hilbert spaces $\left(\mathcal{K},\langle,\rangle_{1}\right)$ with unitary dynamics $C(t)$ and with the inner product satisfying (1.8) or (1.9). On $\mathfrak{V}$, there must exist a complex structure $J$, a real linear operator satisfying $J^{2}=-I$. Since $\langle\zeta, \xi\rangle_{1}$ must be a $J$-sesquilinear form,

$$
\langle J \zeta, J \xi\rangle_{1}=\langle\zeta, \xi\rangle_{1},
$$

which implies that $J$ must be symplectic in the Boson case and orthogonal in the Fermion case. The $J$-sesquilinearity condition $\langle\xi, J \zeta\rangle_{1}=i\langle\xi, \zeta\rangle_{1}$ then determines $\langle\xi, \zeta\rangle_{1}$ uniquely as

$$
\begin{array}{lr}
\langle\xi, \zeta\rangle_{1}=-\alpha^{-2} B(\xi, J \zeta)-\alpha^{-2} i B(\xi, \zeta) & \text { (Boson case) } \\
\langle\xi, \zeta\rangle_{1}=\alpha^{-2} S(\xi, \zeta)-a^{-2} i S(\xi, J \zeta) & \text { (Fermion case) }
\end{array}
$$

In order that the above sesquilinear form extends to a positive definite inner product, it is necessary that $-\alpha B(\xi, J \zeta)$ is strictly positive (Boson case) or that $\alpha>0$ (Fermion case). We require that $C(t)$ be a one parameter group of unitary transformations on $\mathcal{H}=\left(\mathcal{V}, J,\langle\cdot, \cdot\rangle_{1}\right)$. This condition reduces to $[C(t), J]=0$.

Given that in $\mathcal{V}$, there are no non-trivial invariant vectors $v$ such that $C(t) v=v$ and that $C(t)$ has a positive generator on $\mathcal{K}$, the uniqueness of $J$, when it exists, is known from the work of Weinless [25]. For the Boson case, this result was made more explicit by Kay [14]. More recently, Gallone and Sparzani [11] have proven that if there exists any group $\mathbb{B}=\{C(g) ; g \in G\}$ of symplectic transformations which has an irreducible representation on the real space $\mathcal{V}$ such that $C(g)$ are unitary on complex space $\mathcal{H}=\left(\mathfrak{V}, J,\langle\cdot, \cdot\rangle_{1}\right)$, then $J$ is unique. For example, the real solution space $\mathcal{V}$ of the Klein-Gordon equation $\left(\partial_{t}^{2}-\nabla^{2}\right.$ $\left.+m^{2}\right) \phi(\mathbf{x}, t)=0$ carries an irreducible unitarizable representation of the inhomogeneous restricted Lorentz group. Therefore, the unitarization can be effected 
by only one complex structure $J$ on $\mathfrak{W}$. The existence of $J$ for the Klein-Gordon system is related to the unambiguous separation of $\mathfrak{V}$ into particle solutions and anti-particle solutions [22]. However, this separation is no longer possible in the solution space of

$$
\left[\partial_{t}^{2}-\nabla^{2}+m(m-v(x))\right]_{\phi}=0,
$$

when $v(x)$ is allowed to exceed $m$. This so-called Klein paradox and its relationship with the Schiff-Snyder-Weinberg instability phenomenon is discussed by Fulling [9]. Indeed, for the closely related imaginary mass Klein-Gordon equation for the Sudarshan tachyon field, unitarization has long been known to be impossible [25]. Although the existence or non-existence of $J$ has been settled for isolated cases, the existence problem still requires a general resolution, as pointed out quite recently by Gallone and Sparzani [11]. The aim of this paper is to peg back the current lead of uniqueness results over existence results. To this end, we first discuss, in Section 2, the problem for finite degrees of freedom. This restriction has previously enabled a complete characterization of the classical dynamical systems which are and are not unitarizable [4]. When $J$ is unique, an expression is derived for it which is extendible to infinite degrees of freedom. However, to make this extension, we find it necessary to use recent results on spectral theory in indefinite inner product spaces. These are discussed in Section 3. In Section 4, we assume that $\mathfrak{V}$ is a real Hilbert space $(\mathcal{V}, S)$. In the Boson case, the symplectic form $B(\zeta, \xi)$ is assumed to be $S(\zeta, G \xi)$, where $G$ is some fixed real skew symmetric complex structure. The quantity $J$ (Boson case) is then shown to exist whenever $C(t)$ is generated by a strictly positive classical Hamiltonian. We have recently learnt that this very result has been proven independently by Paneitz [18], who stressed the relationship between this work and Krein stability theory, rather than spectral theory in Krein space. Then $J$ (Fermion) exists for any continuous real orthogonal group $C(t)$. However, in Section 5 , we prove that $J$ (Boson case) does not exist unless $C(t)$ is similar to a one parameter real orthogonal group on $(\mathfrak{V}, S)$. This excludes systems such as the imaginary mass Klein-Gordon field which have non-real frequency spectra and also excludes systems which have real frequency spectra but are not diagonalizable, due to non-trivial Jordan blocks.

\section{Quantization of finite classical linear systems}

When $\operatorname{dim} \mathscr{V}<\infty$, a suitable change of basis reduces an inner product $S(\zeta, \xi)$ to the Euclidean inner product $(\zeta, \xi)$ and a symplectic form $B(\zeta, \xi)$ reduces to $(\zeta, G \xi)$ with $G=\left[\begin{array}{cc}0 & -I \\ I & 0\end{array}\right]$. 
Given a classical quadratic Hamiltonian $H=\frac{1}{2}(\mathbf{z}, \hat{H} \mathbf{z})$ with $\hat{H}$ real symmetric and $z=\left[\begin{array}{l}q \\ \mathbf{q}\end{array}\right]$, the Hamiltonian equations yield

$$
\mathbf{z}(t)=\exp [-G \hat{H} t] \mathbf{z}(0) .
$$

Then $C(t)=\exp [-G \hat{H} t]$ is a one parameter group of symplectic transformations. For a review of such classical systems and their quantization, the reader is referred to [3].

Analogously, the classical analogue of a quadratic Fermion Hamiltonian is a quadratic element of a Grassmann algebra, which leads to a one parameter group of orthogonal transformations, via symmetric Poisson bracket equations, as described in [5]. The Segal quantization then begins with the dynamics on real space $\mathcal{V}$

$$
\zeta(t)=\exp [-\hat{A} t] \zeta(0),
$$

where $\hat{A}$ is real skew symmetric (Fermion case) or an element of the symplectic Lie algebra (Boson case). The unitarizing complex structure $J$, a linear operator on real space $\mathfrak{T}$, must satisfy

$$
\begin{aligned}
& J^{2}=-I, \\
& J^{t} G J=G \quad(J \text { is symplectic in the Boson case }), \\
& \text { or } J^{t} J=I \quad(J \text { is orthogonal in the Fermion case), } \\
& {[J, \hat{A}]=0 \quad \text { (due to unitarity of } C(t) \text { in }\left(\mathcal{V}, J,\langle\cdot, \cdot\rangle_{1}\right) \text { ), }} \\
& -G J>0 \quad \text { (positivity of }\langle\cdot, \cdot\rangle_{1} \text { in the Boson case), }
\end{aligned}
$$

or $-J \hat{A}>0$ (positivity of the quantized Hamiltonian in the Fermion case).

In an ordinary Hamiltonian system of one classical degree of freedom, $J \in$ $\operatorname{Sp}(2, R)=S L(2, R)$ and $J^{2}=-I$.

Therefore,

$$
J=\left[\begin{array}{cc} 
\pm(a b-1)^{1 / 2} & b \\
-a & \mp(a b-1)^{1 / 2}
\end{array}\right] \text { for some } a, b \in R .
$$

Since $J$ in this case is specified by only two real parameters, it is easy to determine the commutant of $\hat{A}$, to which $J$ must belong, by (2.3c). It turns out that $\hat{H}$ $(=-G \hat{A})$ must either be trivial $(\hat{H}=0)$ or definite $(\hat{H}>0$ or $\hat{H}<0)$. In these latter cases, the Hamiltonian $H$ can be transformed by a symplectic transformation to that of a harmonic oscillator, $H= \pm \frac{1}{2}\left(p^{2}+\omega^{2} q^{2}\right)$. Evidently, the other one dimensional systems, which must lie in the same canonical class as either the free particle with semi-definite Hamiltonian $H= \pm \frac{1}{2} p^{2}$, or the repulsive "oscillator" with indefinite Hamiltonian $H=\frac{1}{2}\left(p^{2}-\omega^{2} q^{2}\right)$, cannot be unitarized. This was first exhibited by Sparzani and Gallone [10]. When considering arbitrary 
finite degrees of freedom, extra complexity arises because the frequencies (eigenvalues of $i G \hat{H}$ ) may be mixed complex, not just pure imaginary as for the repulsive "oscillator", and even if the frequencies are real, $i G \hat{H}$ may not necessarily have a complete set of eigenvectors, due to non-trivial Jordan canonical blocks. In [4], it was proved that $J$ exists if and only if all frequencies are real and all elementary divisors $\left(s-s_{j}\right)^{N_{j}}$ of $(i G \hat{H}-s I)$ are linear $\left(N_{j}=1\right)$. This means that the whole system can be reduced, by a symplectic transformation, to a collection of independent trivial subsystems and independent harmonic oscillators. In the Fermion case, $i \hat{A}$ is always Hermitian and, as a result, $J$ always exists. When $\operatorname{dim} \operatorname{ker} \hat{A}=0, J$ is unique and is given by:

$$
J=C G C^{-1}, \quad \text { (Boson case), }
$$

where $C$ is a real symplectic transformation (given explicitly in [4]) such that $C^{t} \hat{H} C$ is diagonal, or

$$
J=C G C^{-1} \quad \text { (Fermion case), }
$$

where $C$ is a real orthogonal transformation (given explicitly in [5]) such that

$$
C^{-1} \hat{A} C=\hat{A}_{0},
$$

with $\hat{A}_{0}$ in canonical form

$$
\hat{A_{0}}=\left[\begin{array}{ll|ll} 
& & -s_{1} & \\
& & -s_{m} \\
\hline s_{1} & &
\end{array}\right], \quad s_{J} \in R .
$$

At this point, the reader should be convinced that the technology for constructing $J$ is available in finite degrees of freedom. However, from the outset, one of the aims of Segal's programme was to rigorize the quantization of systems with infinite degrees of freedom. We can now give an equivalent prescription for (2.6) which constructs $J$ more directly from $\hat{A}$ and can be generalized to infinite degrees of freedom. Namely, $J$ is the orthogonal component in the polar decomposition of $\hat{A}$ :

$$
\begin{aligned}
J & =\left[\hat{A}^{t} \hat{A}\right]^{-1 / 2} \hat{A} \\
& =\left[-\hat{A}^{2}\right]^{-1 / 2} \hat{A} \\
& =\left[-C \hat{A}_{0}^{2} C^{-1}\right]^{-1 / 2} C \hat{A}_{0} C^{-1} \quad(\text { from } 2.7) \\
& =C \operatorname{diag}\left[\left|s_{1}\right|^{-1}, \ldots,\left|s_{m}\right|^{-1},\left|s_{1}\right|^{-1}, \ldots,\left|s_{m}\right|^{-1}\right] \hat{A}_{0} C^{-1} \\
& =C G C^{-1}, \quad \text { in agreement with }(2.6) .
\end{aligned}
$$


When $\hat{H}>0$, Whittaker's theorem [26] enables us to find a symplectic transformation $C$ such that

$$
C^{t} \hat{H} C=\hat{H}_{0}=\operatorname{diag}\left[s_{1}, s_{2}, \ldots, s_{N}, s_{1}, \ldots, s_{N}\right], \quad \text { with } s_{J}>0 .
$$

We then rederive (2.10) in the Boson case:

$$
\begin{aligned}
J & =\left[-\hat{A}^{2}\right]^{-1 / 2} \hat{A}=[-G \hat{H} G \hat{H}]^{-1 / 2} G \hat{H} \\
& =\left[-G C^{t^{-1}} \hat{H}_{0} C^{-1} G C^{t^{-1}} \hat{H}_{0} C^{-1}\right]^{-1 / 2} G C^{t^{-1}} \hat{H}_{0} C^{-1} \quad(\text { by } 2.11) \\
& =\left[-C G \hat{H}_{0} C^{-1} C G \hat{H}_{0} C^{-1}\right]^{-1 / 2} C G \hat{H}_{0} C^{-1} \quad\left(\text { since } C, C^{T} \in \operatorname{Sp}(2 N, R)\right) \\
& =C \operatorname{diag}\left[\left|s_{1}\right|^{-1}, \ldots,\left|s_{N}\right|^{-1},\left|s_{1}\right|^{-1}, \ldots,\left|s_{N}\right|^{-1}\right] \hat{A}_{0} C^{-1} \\
& =C G C^{-1}, \quad \text { in agreement with }(2.5) .
\end{aligned}
$$

The expression (2.9') for $J$ is not simply the orthogonal component in the polar decomposition of $\hat{A}$, as in the Fermion case, since

$$
\hat{A}^{t} \hat{A}=(G \hat{H})^{t} G \hat{H}=\hat{H}^{t} G^{t} G \hat{H}=\hat{H}^{2},
$$

which is not necessarily equal to- $(G \hat{H})^{2}$, since $\hat{H}$ does not necessarily commute with $G$. However, $i G \hat{H}$ is self adjoint with respect to the indefinite metric $(\cdot, i G \cdot)$. Therefore,

$$
-(G \hat{H})^{2}=(i G \hat{H})^{+} i G \hat{H},
$$

where $\hat{A}^{+}$denotes the adjoint $-G \hat{A}^{\prime} G$ of $\hat{A}$, with respect to the indefinite metric. Hence, we recover an expression similar to (2.9).

A symplectic transformation $C$ may be viewed as a pseudo-unitary transformation on the Krein space $(\mathcal{V},(\cdot, i G \cdot))$ with indefinite metric. This enables the above-mentioned theorem of Whittaker to be interpreted as a spectral theorem in Krein space [6]. A spectral theory is now available for unbounded operators on infinite dimensional Krein spaces and this enables us to extend Whittaker's theorem to infinite degrees of freedom. Since the notion of polar decomposition of an operator on Hilbert space is well established, (2.9) will be directly applicable to infinite degrees of freedom and after some preliminary ground-work in Section 3 , so too will $\left(2.9^{\prime}\right)$. In fact, the construction $\left(2.9^{\prime}\right)$ was derived by Ashtekar and Magnon [1] for the infinite dimensional system consisting of the Klein-Gordon equation in stationary curved space-time. They arrived at this result via a different route, by imposing that with the system in a single particle state, the expectation value of the Hamiltonian should be equal to the value of the classical Hamiltonian. This imposition, in the situation of finite degrees of freedom, yields

$$
\begin{aligned}
H & =\frac{1}{2}(\mathbf{z}, \hat{H} \mathbf{z})=\langle\mathbf{z},-J G \hat{H} \mathbf{z}\rangle_{1} \\
& =\alpha^{-2}(\mathbf{z}, \hat{H} \mathbf{z})+\alpha^{-2} i(\mathbf{z}, G J G \hat{H} \mathbf{z}) \quad \text { by (1.11a). }
\end{aligned}
$$


Since

$$
\begin{aligned}
(G J G \hat{H})^{\prime} & =\hat{H} G J^{\prime} G \\
& =\hat{H} J \quad(\text { since } J \text { is symplectic }) \\
& =-G J G \hat{H} \quad(\text { since }[J, G \hat{H}]=0),
\end{aligned}
$$

the imaginary part in the right hand side of (2.12) vanishes and (2.12) forces the normalization constant $\alpha$ to be $\sqrt{2}$.

\section{Spectral theory in Krein space}

Suppose that $\left(\mathcal{K}_{c},\langle\cdot, \cdot\rangle\right)$ is a complex Hilbert space and that $\eta$ is a self-adjoint involution on $\mathcal{H}_{c}$,

$$
\eta^{\dagger}=\eta=\eta^{-1}
$$

Then $\eta$ is the metric or Gram operator for a particular indefinite inner product $[\cdot, \cdot]$ on $\mathcal{K}_{c}$.

$$
[\zeta, \xi]=\langle\zeta, \eta \xi\rangle
$$

DEFINITION 3.3.

(a) $A$ densely defined operator $M$ on $\mathcal{H}_{c}$ has $\eta$-adjoint $M^{+}$defined by $M^{+} \phi=\psi$, provided $[\phi, M \chi]=[\psi, \chi]$ for all $\chi \in \operatorname{dom} M$;

(b) $M$ is self $\eta$-adjoint if $M^{+}=M$;

(c) a self $\eta$-adjoint operator $M$ is $\eta$-non-negative if $[\psi, M \psi] \geqslant 0$ for all $\psi \in$ dom $M$.

If, in addition, $[\psi, M \psi]=0 \Rightarrow \psi=0$, then $M$ is strictly $\eta$-positive.

It is easily verified that

$$
M^{+}=\eta M^{\dagger} \eta
$$

$M$ is self $\eta$-adjoint if and only if $M=\eta D$,

where $D$ is self adjoint on $\mathcal{K}_{c}$,

$M$ is $\eta$-non-negative if and only if $D$ given in (b) is non-negative,

$M$ is strictly $\eta$-positive if and only if $D$ is strictly positive.

DEFINITION 3.5. $E$ is an $\eta$-orthogonal projector on $\mathcal{H}_{c}$ if $E$ is self $\eta$-adjoint with domain $\mathcal{K}_{c}$ and $E$ is idempotent, $E^{2}=E$.

If $E$ is an $\eta$-orthogonal projector on $\mathcal{K}_{c}$, then

$$
\forall \phi, \psi \in \mathcal{K}_{c}, \quad[E \phi,(1-E) \psi]=0 .
$$


Definition 3.6. An $\eta$-spectral function defined on the interval $(a, b)$ with $a<0<b$, is an operator valued function $E(t)$ on $(a, b)$ satisfying

(a) $E(t)$ is an $\eta$-orthogonal projector on $\mathcal{K}_{c}$.

(b) $E(s) E(t)=E(\min \{s, t\})$.

(c) In the strong operator topology,

$$
\lim _{t \rightarrow s^{+}} E(t)=E(s) \quad \text { for all } s \in(a, b),
$$

and

(d) In the strong operator topology,

$$
\lim _{t \rightarrow b^{-}} E(t)=I \text { and } \quad \lim _{t \rightarrow a^{+}} E(t)=0 .
$$

THEOREM 3.7. Let $M$ be a densely defined strictly $\eta$-positive operator whose resolvent set $\rho(M)$ is non-empty. Then $\mathcal{H}_{c}$ is a direct sum of M-invariant $\eta$-orthogonal subspaces $\mathcal{H}_{1}$ and $\mathcal{H}_{2}$ such that

(a) $M_{1}=M / \mathcal{K}_{1}=\int_{-\infty}^{\infty} t d E_{1}(t)$ (converging in the strong topology) for some $\eta$-spectral function $E_{1}(t)$.

(b) For all $\phi \in \mathcal{H}_{2} \cap \operatorname{dom} M, M \phi=\int_{-\infty}^{\infty} t^{-1} d E_{2}(t) \phi$ (converging in the strong topology) for some $\eta$-spectral function $E_{2}(t)$.

(c) $[\psi, \pm \psi]$ is positive definite on $E_{J}(t) \mathcal{H}$, for $t \gtrless 0$.

For bounded operators $M, \mathcal{H}_{2}$ may be neglected, and in this case, the above spectral theorem was founded by Krein and Smul'jan [15]. A clear discussion of this subject, including the uniqueness of $E_{1}(t)$, may be found in [2]. The extension to unbounded $M$ has recently been achieved by Harvey [13]. In fact, the spectral theorem is established for $\eta$-non-negative operators $M$, but the loss of strict positivity introduces extra complications which are not applicable to the task which we have in mind.

For physical applications, it has been convenient, following Dirac [8], to interpret the spectral decomposition of an unbounded normal operator $Q$ on Hilbert space as the existence of a complete orthonormal set of eigenfunctions. In some circumstances, distribution theory rigorously justifies this notion. In particular, by Theorem 6 in Chapter II of [16], if there exists a dense space $\delta$ of $C_{\infty}$ vectors for $M\left(\delta=\bigcap_{n \in N}\right.$ dom $Q^{n}$, with $N$ the set of natural numbers), then there exists a nuclear space $\mathscr{T}$ which is dense in $\mathcal{S}$ and $\mathcal{K}_{c}$ can be continuously embedded in the rigged Hilbert space consisting of Gelfand triplet $\Re \subset \mathcal{H}_{c} \subset \Re^{\prime}$, with the dual space $\Re^{\prime}$ being the space of continuous linear functionals on $\Re$. It then follows (Theorem 5 in Chapter 11 of [16]) that there exists in $\Re^{\prime}$ a complete orthonormal set of eigendistributions $\psi_{\alpha}$ for $Q$. A formal expansion

$$
\mathcal{H} \ni \psi=\int_{S} \psi_{\alpha} a(\alpha) d \sigma(\alpha),
$$


with $S$ a set of spectral indices and $\sigma$ an appropriate measure, has the following meaning:

$$
\text { if } \phi \in \mathscr{N}, \psi(\phi)=(\psi, \phi)=\int_{S} \psi_{\alpha}(\phi) a(\alpha) d \sigma(\alpha)
$$

With intuitive physical applications in mind, we now reformulate Theorem 3.7 in terms of eigendistributions.

THEOREM 3.9. Let $M$ be as specified in Theorem 3.7, with the additional property that $M$ has a dense set of $C_{\infty}$ vectors in $\mathcal{K}_{c}$. Then there exists a dense nuclear space $\mathfrak{X} \subset \mathfrak{K}_{c}$ such that $\mathfrak{X}^{\prime}$ contains a complete $\eta$-orthonormal set of eigendistributions $\psi_{\alpha}$ for $M$.

Proof. Define

$$
\mathscr{V}_{+}=\left(I-E_{1}(0)\right) \mathcal{H}_{1} \cup\left(I-E_{2}(0)\right) \mathcal{H}_{2}
$$

and

$$
\mathfrak{V}_{-}=E(0) \mathcal{K}_{1} \cup E(0) \mathcal{K}_{2}
$$

By $(3.7 \mathrm{c})$, the inner product $\langle\phi, \pm \eta \phi\rangle$ is positive definite on $\mathcal{V}_{ \pm}$. Therefore, $\left(\mathscr{V}_{ \pm},\langle\cdot, \pm \eta \cdot\rangle\right)$ may be completed to form Hilbert spaces $\mathcal{K}_{ \pm}$, on which the corresponding restrictions $M_{ \pm}$of $M$ are self adjoint. Hence, we may apply the spectral theory in ordinary Hilbert space. Let $\chi$ be an arbitrary $\mathcal{C}_{\infty}$ vector $(\chi \in \mathfrak{S})$ for $M$. Clearly, $E(0) \chi \in \delta$. Therefore, $E(0) \Im \subset \delta \cap \mathcal{H}_{-}$. To show that $E(0) \mathcal{S}$ is dense in $\mathcal{K}_{-}$, let $\psi$ be an arbitrary element of $\mathfrak{V}_{-} \subset \mathcal{K}_{c}$. Since $\delta$ is dense in $\mathcal{K}_{c}$, there exists a sequence $\left\{\phi_{n}\right\}$ in $\delta$ such that

$$
\begin{aligned}
& \left\langle\psi-\phi_{n}, \psi-\phi_{n}\right\rangle \rightarrow 0 \\
& \quad \Rightarrow\left\langle E(0)\left(\psi-\phi_{n}\right), E(0)\left(\psi-\phi_{n}\right)\right\rangle \rightarrow 0 \quad(\text { since } E(0) \text { is a bounded projector }) \\
& \quad \Rightarrow\left\langle\psi-E(0) \phi_{n}, \psi-E(0) \phi_{n}\right\rangle \rightarrow 0 \quad\left(\text { since } E(0) \psi=\psi \in \mathcal{V}_{-}\right) .
\end{aligned}
$$

Therefore, $E(0) \mathcal{S}$ is dense in $\mathcal{V}_{-}$, which is dense in $\mathcal{H}_{-}$. The set $\mathcal{S}_{-}$of $\mathcal{C}_{\infty}$ vectors for $M_{-}$, which contains $E(0) \mathcal{S}$, is dense in $\mathscr{K}_{-}$. Therefore, there exists in $\mathscr{K}_{-}$a dense $M$-invariant nuclear space $\Re_{-}$. Similarly, there exists in $\mathcal{K}_{+}$a dense $M$-invariant nuclear space $\Re_{+}$. Hence, there exists in the dual spaces $\mathfrak{K}_{ \pm}^{\prime}$ a complete $\eta$-orthonormal system of eigendistributions of $M_{ \pm}$. These eigendistributions $\psi_{\mathbf{k}, 1}^{( \pm)}$are considered to be labelled by the discrete spectral values $\mathbf{k}$ and 
continuous spectral values $I$ of a complete set of commuting self-adjoint operators $\left\{K_{1}^{ \pm}, K_{2}^{ \pm}, \ldots ; L_{1}^{( \pm)}, L_{2}^{( \pm)}, \ldots\right\}$

$$
\begin{gathered}
M \psi_{\mathbf{k}, \mathbf{l}}^{( \pm)}=m^{ \pm}(\mathbf{k}, \mathbf{l}) \psi_{\mathbf{k}, \mathbf{l}}^{( \pm)} \quad \text { with } m^{ \pm}(\mathbf{k}, \mathbf{l}) \gtrless 0, \\
0=\left\langle\psi_{\left.\mathbf{k}, \mathbf{l}^{(}\right)}^{(+)}, \eta \psi_{\mathbf{k}^{\prime}, \mathbf{l}^{\prime}}^{(-)}\right\rangle, \\
\delta_{\mathbf{k}, \mathbf{k}^{\prime}} \delta\left(\mathbf{l}-\mathbf{l}^{\prime}\right)=\left\langle\psi_{\mathbf{k}, \mathbf{l}}^{(+)}, \eta \psi_{\mathbf{k}^{\prime}, l^{\prime}}^{(+)}\right\rangle=-\left\langle\psi_{\mathbf{k}, \mathbf{l}}^{(-)}, \eta \psi_{\mathbf{k}^{\prime}, \mathbf{l}^{\prime}}^{(-)}\right\rangle .
\end{gathered}
$$

In the applications to classical mechanics, we are concerned with real symplectic transformations on a real classical space. We need to modify the above spectral theory for complex Krein space to accommodate a real symplectic space. The following theorem achieves the simultaneous canonical reduction of a strictly positive definite real self adjoint operator and a real skew symmetric complex structure.

THEOREM 3.12. Let $(\mathcal{K},(\cdot, \cdot))$ be a real separable Hilbert space, $G$ be a skew adjoint complex structure on $\mathcal{H}$ and $\hat{H}$ be a self adjoint operator on $\mathcal{H}$ such that

(a) $\exists \varepsilon>0, \hat{H}-\varepsilon I$ is strictly positive;

(b) the set $\delta$ of $C_{\infty}$ vectors for $G \hat{H}$ is dense in $\mathcal{H}$.

Then there exists a dense nuclear space $\Re \subset \mathcal{H}$ such that the dual $\Re$ ' of $\mathscr{\Re}$ contains a basis $\left\{\psi_{\alpha} ; \alpha \in S\right\} \cup\left\{\psi_{\alpha}^{\prime} ; \alpha \in S\right\}$ ( $S$ may be considered to be a set of spectral indices) in which simultaneously $G=\left[\begin{array}{cc}0 & -I \\ 1 & 0\end{array}\right]$ and $\hat{H}=\left[\begin{array}{cc}D & 0 \\ 0 & D\end{array}\right]$, with $D$ positive definite diagonal.

Proof. Define a complex structure $K=\left[\begin{array}{cc}0 & -I \\ 1 & 0\end{array}\right]$ on $\mathcal{K} \oplus \mathcal{H}$.

The usual inner product $\left(\psi_{1}, \phi_{1}\right)+\left(\psi_{2}, \phi_{2}\right)$ between elements $\Psi=\psi_{1} \oplus \psi_{2}$ and $\Phi=\phi_{1} \oplus \phi_{2}$ will be denoted $(\Psi, \Phi)$. Define a $K$-sesquilinear form

$$
\langle\Psi, \Phi\rangle=(\Psi, \Phi)-i(\Psi, K \Phi),
$$

Then $\mathcal{C}=\left[\begin{array}{cc}I & 0 \\ 0-I\end{array}\right]$ is a $K$-conjugation, since

$$
\bigodot(a+b K) \Psi=a \bigodot \Psi-b K \bigodot \Psi \quad \text { for all } a, b \in R .
$$

Since $\langle\Psi, \Phi\rangle=(\Psi, \Phi)$, the elements of $\mathcal{K} \oplus \mathcal{K}$, furnished with complex structure $K$ and inner product $\langle\cdot, \cdot\rangle$, constitute a complex Hilbert space $\mathcal{H}^{c}$.

In the following, if $A$ is an operator on $\mathcal{H}, A^{(2)}$ will denote $A \oplus A$. The involution $\eta=K G^{(2)}=\left[\begin{array}{cc}0 & -G \\ G & 0\end{array}\right]$ is self-adjoint on $\mathcal{H}^{c}$. Define a strictly $\eta$-positive operator $M=\eta \hat{H}^{(2)}$. We shall verify that $M$ satisfies all requirements given in Theorem 3.9.

$$
M^{\dagger} M=\hat{H}^{(2)^{2}}=\hat{H}^{2} \oplus \hat{H}^{2}>\varepsilon^{2} I^{(2)},
$$


which implies that $\rho(M)$ is non-empty. Clearly, $\delta \oplus \delta$ is dense in $\mathcal{K}^{c}$. To show that each element $\phi_{1} \oplus \phi_{2} \in \mathcal{S} \oplus \mathcal{S}$ is a $C_{\infty}$ vector for $M$,

$$
\begin{aligned}
\left\langle M^{n} \Phi, M^{n} \Phi\right\rangle= & \left\langle K^{n}\left(G^{(2)} \hat{H}^{(2)}\right)^{n} \Phi, K^{n}\left(G^{(2)} \hat{H}^{(2)}\right)^{n} \Phi\right\rangle \\
& \quad\left(\text { since } K \text { commutes with } G^{(2)} \hat{H}^{(2)}\right) \\
= & \left.\left\langle\left(G^{(2)} \hat{H}^{(2)}\right)^{n} \Phi,\left(G^{(2)} \hat{H}^{(2)}\right)^{n} \Phi\right\rangle \text { (since } K \text { is unitary on } \mathcal{H}^{c}\right) \\
= & \left((G \hat{H})^{n} \phi_{1},(G \hat{H})^{n} \phi_{1}\right)+\left((G \hat{H})^{n} \phi_{2},(G \hat{H})^{n} \phi_{2}\right)<\infty,
\end{aligned}
$$

since $\phi_{1}$ and $\phi_{2}$ are $\mathcal{C}_{\infty}$ vectors for $G \hat{H}$.

By Theorem (3.9), there exist $\eta$-orthogonal subspaces $\mathcal{H}_{ \pm}^{c}$ such that

(1) $\langle\cdot, \pm \eta \cdot\rangle$ is positive definite on $\mathcal{K}_{ \pm}^{c}$.

(2) There exist dense nuclear spaces $\Re_{ \pm} \subset \mathcal{H}_{ \pm}^{c}$ such that the dual spaces $\Re_{ \pm}^{\prime}$ contain bases $\left\{\Psi_{\mathrm{k} .1}^{( \pm)}\right\}$of eigendistributions of $M$ satisfying

$$
\begin{aligned}
& M \Psi_{\mathrm{k}, \mathrm{I}}^{( \pm)}=m^{ \pm}(\mathbf{k}, \mathbf{l}) \Psi_{\mathrm{k}, \mathrm{I}}^{ \pm} \quad \text { with } m^{ \pm} \gtrless 0, \\
& \pm\left\langle\Psi_{\mathbf{k}, l^{\prime}}^{( \pm)}, \eta \Psi_{\mathbf{k}^{\prime}, \mathbf{l}^{\prime}}^{( \pm)}\right\rangle=\delta_{\mathbf{k}^{\prime}, \mathbf{k}^{\prime}} \delta\left(\mathbf{l}-\mathbf{l}^{\prime}\right), \\
& \left\langle\Psi_{\mathbf{k}, \mathbf{l}}^{(+)}, \eta \Psi_{\mathbf{k}^{\prime}, \mathbf{l}^{\prime}}^{(-)}\right\rangle=0 \text {. }
\end{aligned}
$$

Now

$$
M \mathcal{C} \Psi_{\mathbf{k}, \mathbf{l}}^{(+)}=-\mathcal{M} \Psi_{\mathbf{k}, \mathbf{l}}^{(+)}=-m^{+}(\mathbf{k}, l) \mathcal{C} \Psi_{\mathbf{k}, \mathbf{l}}^{(+)}
$$

Hence, $\Psi_{k, 1}^{(+)}$is an eigendistribution of $M$ corresponding to spectral value $m^{+}$if and only if $C \Psi_{k, 1}^{(+)}$is an eigendistribution corresponding to spectral value $-m^{+}$.

In addition,

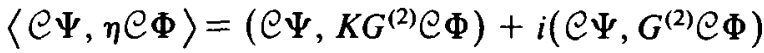

$$
\begin{aligned}
& =-\left(\Psi, K G^{(2)} \Phi\right)-i\left(\Psi, G^{(2)} \Phi\right) \\
& =-\langle\Psi, \eta \Phi\rangle \text {. }
\end{aligned}
$$

Therefore, we may take the bases of eigendistributions to be $\left\{\Psi_{k, 1}^{(+)}\right\}$and $\left\{\mathcal{C} \Psi_{k, 1}^{(+)}\right\}$.

From the basis distributions

$$
\Psi_{k, 1}=\psi_{k, 1} \oplus \psi_{k, 1}^{\prime}
$$

and

$$
\bigodot \Psi_{\mathbf{k}, \mathbf{l}}=\psi_{\mathbf{k}, \mathbf{l}} \oplus-\psi_{\mathbf{k}, \mathbf{l}}^{\prime}
$$

let us construct

$$
X_{k, 1}=\frac{1}{2}\left(\Psi_{k, 1}+C \Psi_{k, 1}\right)=\psi_{k, 1} \oplus 0
$$

and

$$
\Xi_{\mathbf{k}, 1}=\frac{1}{2} K\left(\Psi_{\mathbf{k}, 1}-e \Psi_{\mathbf{k}, 1}\right)=-\psi_{\mathbf{k}, 1}^{\prime} \oplus \mathbf{0}
$$


Since $\left\{\Psi_{k, 1}, \mathcal{C} \Psi_{k, 1}\right\}$ is a basis for $\mathcal{H}^{c}$, every element $\Psi=\psi \oplus \psi^{\prime}$ being expanded as

$$
\begin{aligned}
\psi \oplus \psi^{\prime}= & \Psi=\int[a(\mathbf{k}, \mathbf{l})+b(\mathbf{k}, \mathbf{l}) K] \psi_{\mathbf{k}, \mathbf{l}} \\
& \quad+\left[a^{\prime}(\mathbf{k}, \mathbf{l})+b^{\prime}(\mathbf{k}, \mathbf{l}) K\right] \mathcal{C} \Psi_{\mathbf{k}, \mathbf{l}} d \sigma(\mathbf{k}, \mathbf{l}) \quad\left(a, b, a^{\prime}, b^{\prime} \in R\right) \\
= & \int\left[\left(a+a^{\prime}\right)+\left(b+b^{\prime}\right) K\right] X_{\mathbf{k}, \mathbf{l}}+\left[\left(b-b^{\prime}\right)+\left(a-a^{\prime}\right) K\right] \Xi_{\mathbf{k}, \mathbf{l}} d \sigma \\
= & \int\left[a(\mathbf{k}, \mathbf{l})+a^{\prime}(\mathbf{k}, \mathbf{l})\right] \psi_{\mathbf{k}, \mathbf{l}}+\left[b^{\prime}(\mathbf{k}, \mathbf{l})-b(\mathbf{k}, \mathbf{l})\right] \psi_{\mathbf{k}, \mathbf{l}}^{\prime} d \sigma \\
& \oplus \int\left[b(\mathbf{k}, \mathbf{l})+b^{\prime}(\mathbf{k}, \mathbf{l})\right] \psi_{\mathbf{k}, \mathbf{l}}+\left[a^{\prime}(\mathbf{k}, \mathbf{l})-a(\mathbf{k}, \mathbf{l})\right] \psi_{\mathbf{k}, \mathbf{l}}^{\prime} d \mathbf{\sigma},
\end{aligned}
$$

$\left\{\psi_{\mathrm{k}, \mathrm{l}}, \psi_{\mathrm{k}, \mathrm{l}}^{\prime}\right\}$ is evidently a basis for the real Hilbert space $\mathcal{H}$.

Now

$$
\left\langle X_{\mathbf{k}, 1}, G^{(2)} \Xi_{\mathbf{k}^{\prime}, l^{\prime}}\right\rangle=\frac{1}{2} \delta_{\mathbf{k}, \mathbf{k}^{\prime}} \delta\left(\mathbf{l}-\mathbf{I}^{\prime}\right)
$$

and

$$
\left\langle X_{\mathbf{k}, 1}, G^{(2)} X_{\mathbf{k}^{\prime}, \mathbf{l}^{\prime}}\right\rangle=\left\langle\Xi_{\mathbf{k}, \mathbf{l}}, G^{(2)} \Xi_{\mathbf{k}^{\prime}, \mathbf{l}^{\prime}}\right\rangle=0,
$$

which implies

$$
\left(\psi_{\mathbf{k}, 1}, G \psi_{\mathbf{k}^{\prime}, l^{\prime}}^{\prime}\right)=\left(\psi_{\mathrm{k}, 1}^{\prime}, G \psi_{\mathbf{k}^{\prime}, \mathbf{l}^{\prime}}^{\prime}\right)=0
$$

and

$$
\left(\psi_{\mathbf{k}, \mathbf{l}}, G \psi_{\mathbf{k}^{\prime}, \mathbf{l}^{\prime}}^{\prime}\right)=-\frac{1}{2} \delta_{\mathbf{k}, \mathbf{k}^{\prime}} \delta\left(\mathbf{l}-\mathbf{I}^{\prime}\right)
$$

Also,

$$
\begin{aligned}
\left\langle X_{\mathbf{k}, l}, \hat{H}^{(2)} X_{\mathbf{k}^{\prime}, \mathbf{I}^{\prime}}\right\rangle & =\frac{1}{4}\left\langle\Psi_{\mathbf{k}, \mathbf{l}}^{(+)}+\mathcal{C} \psi_{\mathbf{k}, \mathbf{l}}^{(+)}, \eta M\left(\psi_{\mathbf{k}^{\prime}, I^{\prime}}^{(+)}+\mathcal{C} \Psi_{\mathbf{k}^{\prime}, \mathbf{I}^{\prime}}^{(+)}\right)\right\rangle \\
& =\frac{1}{2} m^{+}(\mathbf{k}, \mathbf{l}) \boldsymbol{\delta}_{\mathbf{k}, \mathbf{k}^{\prime}} \delta\left(\mathbf{I}-\mathbf{I}^{\prime}\right) .
\end{aligned}
$$

This implies

$$
\left(\psi_{\mathbf{k}, \mathbf{l}}, \hat{H} \psi_{\mathbf{k}^{\prime}, \mathbf{l}^{\prime}}\right)=\frac{1}{2} m^{+}(\mathbf{k}, \mathbf{l}) \delta_{\mathbf{k}, \mathbf{k}^{\prime}} \delta\left(\mathbf{l}-\mathbf{l}^{\prime}\right)
$$

Similarly,

$$
\left(\psi_{\mathbf{k}, 1}^{\prime}, \hat{H} \psi_{\mathbf{k}^{\prime}, \mathrm{I}^{\prime}}^{\prime}\right)=\frac{1}{2} m^{+}(\mathbf{k}, \mathbf{l}) \delta_{\mathbf{k}, \mathbf{k}^{\prime}} \delta\left(\mathbf{l}-\mathbf{I}^{\prime}\right)
$$

and

$$
\left(\psi_{\mathbf{k}, 1}, \hat{H} \psi_{\mathbf{k}^{\prime}, \mathbf{l}^{\prime}}^{\prime}\right)=\left(\psi_{\mathbf{k}, 1}^{\prime}, \hat{H} \psi_{\mathbf{k}^{\prime}, \mathbf{l}^{\prime}}\right)=0
$$

By (3.19)-(3.22), the basis of distributions $\left\{2^{1 / 2} \psi_{k, 1}\right\} \cup\left\{2^{1 / 2} \psi_{k, 1}^{\prime}\right\}$ satisfies all the properties required to verify Theorem (3.12). 


\section{Existence results for unitarizing classical dynamics}

In Sections 4 and 5 , the classical real symplectic space $(\mathcal{V}, B)$ which we shall consider, will be assumed to be of a special type. Namely, $\widetilde{V}$ will be a real Hilbert space with inner product $(\zeta, \xi)$ and the symplectic form will be

$$
B(\zeta, \xi)=(\zeta, G \xi)
$$

Whête $G$ is a a predétermined skew adjoint compiex structure on $\tilde{V}$. Ás discussed in Section 2, this assumption can always be made for finite degrees of freedom. In addition, familiar infinite symplectic systems such as the Klein-Gordon system can be integrated into this framework [22].

The dynamics of a classical system may be viewed as a continuous one parameter group of symplectic transformations $C(t)$. If $C(t)$ is strongly continuous then this group of $i G$-unitary transformations has a generator, $C(t)=$ $\exp (-t G \hat{H})$, by an extension of Stone's theorem to indefinite inner product space [17].

THEOREM 4.2. Let $\hat{H}$ be a self adjoint operator on real space $\mathcal{H}$ such that

(1) there exists $\varepsilon>0$ for which $\hat{H}-\varepsilon I>0$, and

(2) there exists in $\hat{H}, a$ dense space of $\bigodot_{\infty}$ vectors for $G \hat{H}$. Then there exists a complex structure $J$, by which the symplectic dynamics, $\zeta(t)=\exp [-G \hat{H} t] \zeta(0)$, may be unitarized.

Proof. Let $M=\eta \hat{H}^{(2)}$, with $\eta=K G^{(2)}$, as in (3.15). Then $M$ satisfies all conditions of Theorem (3.7). With $\mathcal{H}_{j}$ and $E_{j}(t)$ defined as in Theorem (3.7), define a linear operator $J^{(2)}$ by

$$
\mathscr{K}_{j} \ni \phi \rightarrow J^{(2)} \phi=-K\left[1-2 E_{j}(0)\right] \phi ; \quad j=1,2 .
$$

Although $J^{(2)}$ is an operator on the complex space $\mathcal{H}_{c}$, the real space $\mathcal{H} \oplus\{0\} \approx \mathcal{H}$ is $J^{(2)}$-invariant. To see this, recall that Theorem 3.12 establishes a basis of eigendistributions

$$
\left\{X_{\mathbf{k}, \mathbf{l}}=\frac{1}{2}\left(\Psi_{\mathbf{k}, \mathbf{l}}^{(+)}+\mathcal{C} \Psi_{\mathbf{k}, \mathbf{l}}^{(+)}\right)\right\} \cup\left\{\Xi_{\mathbf{k}, \mathbf{l}}=\frac{1}{2} K\left(\Psi_{\mathbf{k}, \mathbf{l}}^{(+)}-\mathcal{C} \Psi_{\mathbf{k}, \mathbf{l}}^{(+)}\right)\right\}
$$

for $\mathcal{K} \oplus\{0\}$.

From the definition of $X$ and $\Xi$, it is easily verified that

$$
J^{(2)} X_{k, 1}=-\Xi_{k, 1}
$$

and

$$
J^{(2)} \Xi_{k, 1}=X_{k, 1}
$$


We may define $J$ to be the restriction of $J^{(2)}$ to $\mathcal{H} \oplus\{0\} \simeq \mathcal{K}$. Clearly

$$
J^{(2)^{2}}=-I^{(2)} \text {, so that } J^{2}=-I \text {. }
$$

Also, $J^{(2)}$ is $\eta$-unitary, since

$$
J^{(2)+} J^{(2)}=\left[1-2 E_{j}(0)\right] K^{+} K\left[1-2 E_{j}(0)\right]=I .
$$

However, $\left\langle\cdot, K G^{(2)} \cdot\right\rangle$ restricted to $\mathcal{K} \oplus 0$ is simply a multiple of the symplectic form on $\mathcal{H}$.

Now,

$$
\begin{aligned}
\left\langle\left[\begin{array}{l}
\phi \\
0
\end{array}\right], K G^{(2)}\left[\begin{array}{l}
\psi \\
0
\end{array}\right]\right\rangle & =\left[\left[\begin{array}{l}
\phi \\
0
\end{array}\right],\left[\begin{array}{cc}
0 & -G \\
G & 0
\end{array}\right]\left[\begin{array}{l}
\psi \\
0
\end{array}\right]\right]+i\left[\left[\begin{array}{l}
\phi \\
0
\end{array}\right],\left[\begin{array}{ll}
G & 0 \\
0 & G
\end{array}\right]\left[\begin{array}{l}
\psi \\
0
\end{array}\right]\right] \\
& =i(\phi, G \psi)=i B(\phi, \psi) .
\end{aligned}
$$

Therefore, from (4.5), $J$ is symplectic on $\mathcal{K}$. To show that $-B(\phi, J \psi)$ is positive definite

$$
-B(\phi, J \psi)=i\left\langle\left(\begin{array}{l}
\phi \\
0
\end{array}\right), K G^{(2)} J^{(2)}\left(\begin{array}{l}
\phi \\
0
\end{array}\right)\right\rangle, \quad \text { by }(4.6) .
$$

Now,

$$
\begin{gathered}
i\left\langle X_{\mathbf{k}, 1}, K G^{(2)} J^{(2)} X_{\mathbf{k}^{\prime}, \mathbf{I}^{\prime}}\right\rangle=-i\left\langle X_{\mathbf{k}, 1}, K G^{(2)} \Xi_{\mathbf{k}^{\prime}, I^{\prime}}\right\rangle, \text { by }(4.3) \\
=\left\langle X_{\mathbf{k}, 1}, G^{(2)} \Xi_{\mathbf{k}^{\prime}, \mathbf{l}^{\prime}}\right\rangle \quad(\text { since }\langle\cdot, \cdot\rangle \text { is a } K \text {-sesquilinear form) } \\
=\frac{1}{2} \boldsymbol{\delta}_{\mathbf{k}, \mathbf{k}^{\prime}} \delta\left(\mathbf{I}-\mathbf{I}^{\prime}\right), \text { by }(3.18) .
\end{gathered}
$$

Similarly,

$$
i\left\langle\Xi_{\mathbf{k}, 1}, K G^{(2)} J^{(2)} \Xi_{\mathbf{k}^{\prime}, \mathbf{I}^{\prime}}\right\rangle=\frac{1}{2} \delta_{\mathbf{k}, \mathbf{k}^{\prime}} \delta\left(\mathbf{l}-\mathbf{l}^{\prime}\right) .
$$

To show that $J$ commutes with $G \hat{H}$,

$$
\begin{aligned}
J^{(2)} G^{(2)} \hat{H}^{(2)} X_{\mathbf{k}, \mathbf{l}} & =-J^{(2)} K M X_{\mathbf{k}, \mathbf{l}} \\
& =-m^{+}(\mathbf{k}, \mathbf{l}) J^{(2)} \Xi_{\mathbf{k}, \mathbf{l}}, \quad \text { by }(3.15) \\
& =-m^{+}(\mathbf{k}, \mathbf{l}) X_{\mathbf{k}, \mathbf{1}}, \quad \text { by }(4.3 \mathrm{~b})
\end{aligned}
$$

and

$$
\begin{aligned}
G^{(2)} \hat{H}^{(2)} J^{(2)} X_{\mathrm{k}, 1} & =-G^{(2)} \hat{H}^{(2)} \Xi_{\mathrm{k}, 1}, \quad \text { by }(4.3 \mathrm{a}) \\
& =K M \Xi_{\mathrm{k}, \mathrm{l}}=m^{+}(\mathbf{k}, 1) K^{2} X_{\mathrm{k}, \mathrm{l}}, \quad \text { by }(3.15) \\
& =-m^{+}(\mathbf{k}, \mathrm{l}) X_{\mathrm{k}, \mathrm{l}}=J^{(2)} G^{(2)} \hat{H}^{(2)} X_{\mathrm{k}, 1}
\end{aligned}
$$

Similarly,

$$
J^{(2)} G^{(2)} \hat{H}^{(2)} \Xi_{k, 1}=G^{(2)} \hat{H}^{(2)} J^{(2)} \Xi_{k, 1}=-m^{+}(\mathbf{k}, 1) \Xi_{k, 1} \text {. }
$$

We have now verified that $J^{(2)}$, restricted to $\mathcal{H} \oplus\{0\}$, satisfies all the properties of a unitarizing complex structure for the symplectic dynamical group $\exp [-G H t]$. 
The above solution for $J$ generalizes that found in Section 2 for finite dimensional systems. The use of complex structure $K$ on $\mathcal{H} \oplus \mathcal{K}$ is equivalent to the extension of $\mathcal{H}$ to the complex field by the formal square root $i$ of -1 . If $\phi_{1}$, $\phi_{2} \in \mathcal{K}_{1,2}$,

$$
\begin{aligned}
& {[-G \hat{H} G \hat{H}]^{-1 / 2} G \hat{H}\left[\phi_{1} \oplus \phi_{2}\right]} \\
& \left.\quad=\left[-M^{2}\right]^{-1 / 2} i M\left[\phi_{1} \oplus \phi_{2}\right] . \quad \text { (where } M=\eta \hat{H}, \eta=i G\right) \\
& \quad=-i \int_{-\infty}^{\infty} \frac{t}{|t|} d E_{1}(t) \phi_{1} \oplus-i \int_{-\infty}^{\infty} \frac{t}{|t|} d E_{2}(t) \phi_{2} \\
& \quad \text { (using spectral theory in Krein space) } \\
& =-i\left[I-2 E_{1}(0)\right] \phi_{1} \oplus-i\left[I-2 E_{2}(0)\right] \phi_{2}, \quad \text { in agreement with (4.2). }
\end{aligned}
$$

In the Fermi-Dirac case, a continuous one parameter group of real orthogonal transformations $C(t)=\exp [-\hat{A} t]$, with the point spectrum of $\hat{A}$ not containing zero, may be unitarized by the complex structure which is the isometric factor in the polar factorization of $\hat{A}$.

$$
J=\left[\hat{A}^{T} \hat{A}\right]^{-1 / 2} \hat{A}=\left[-\hat{A}^{2}\right]^{-1 / 2} \hat{A} .
$$

Since zero is not in the point spectrum of $\hat{A}$, the isometric factor $J$ is in faci orthogonal, as required by Section 1 . From (4.8), it is clear that $[J, \hat{A}]=0$ and $J^{2}=-I$.

$$
-J \hat{A}=\left[\hat{A}^{T} \hat{A}\right]^{-1 / 2} \hat{A}^{T} \hat{A}=\left[\hat{A}^{T} \hat{A}\right]^{1 / 2},
$$

which is clearly positive definite. Therefore, we conclude that (4.8) is the unitarizing complex structure for the classical dynamics generated by $\hat{A}=-\hat{A}^{T}$. By Weinless' result, $J$ is unique, provided $\operatorname{ker} \hat{A}=\{0\}$. In the case that $\operatorname{ker} \hat{A}$ is non-trivial, $J$ still exists and its restriction to $\mathcal{H} \sim \operatorname{ker} \hat{A}$ is given by (4.8), while there is some arbitrariness in the choice of $J / \operatorname{ker} \hat{A}$, due to the triviality of the condition $[J, \hat{A}]=0$.

\section{A non-existence result for unitarizing classical dynamics}

Let $G$ be a distinguished real skew symmetric complex structure on real Hilbert space $(\mathcal{H},(\cdot, \cdot))$. This defines a symplectic form $B(\cdot, \cdot)=(\cdot, G \cdot)$ on $\mathcal{H}$.

In the Segal quantization procedure, complex single particle space $\mathcal{H}_{c}$ is constructed from real symplectic space $\mathscr{R}=(\mathcal{K}, B)$ by choosing a complex structure $J$ on $\mathcal{H}$ so that a prescribed one-parameter group $C(t)$ of symplectic 
transformations on $\mathfrak{T}$ is a strongly continuous group of unitary transformations with respect to the complex inner product

$$
\langle\cdot, \cdot\rangle=-\frac{1}{2} B(\cdot, J \cdot)-\frac{1}{2} i B(\cdot, \cdot) \text {. }
$$

Then, by Stone's theorem, $C(t)=\exp \left(\hat{A}_{c} t\right)$, where $\hat{A}_{c}$ is skew adjoint on $\mathcal{H}_{c}$. The operator $\hat{A}_{c}$ will be denoted by $\hat{A}$ when considered as an operator on the real space $\mathcal{H}$. It will be demonstrated that if $\hat{A}$ is not similar to a real skew adjoint operator on $\mathcal{H}$, then $J$ does not exist and the Segal procedure is not possible. This rules out any unstable classical modes, including those with complex frequency.

Since $\operatorname{dom} J=\mathcal{K}$, we may apply Theorem 3.12 to $-G J$, since $-G J>0$ and $-G J$ has a bounded inverse $-J G$. There exists a basis

$$
\mathscr{P}=\left\{\psi_{\alpha} ; \alpha \in S\right\} \cup\left\{\psi_{\alpha}^{\prime} ; \alpha \in S\right\}
$$

of distributions such that

$$
\begin{gathered}
\left(\psi_{\alpha}^{\prime},-G J \psi_{\beta}\right)=\left(\psi_{\alpha}^{\prime},-G J \psi_{\beta}^{\prime}\right)=d(\alpha) \delta(\alpha-\beta) \quad \text { with } d>0, \\
\left(\psi_{\alpha},-G J \psi_{\beta}^{\prime}\right)=0, \quad\left(\psi_{\alpha}^{\prime}, G \psi_{\beta}\right)=\delta(\alpha-\beta),
\end{gathered}
$$

and

$$
\left(\psi_{\alpha}, G \psi_{\beta}\right)=\left(\psi_{\alpha}^{\prime}, G \psi_{\beta}^{\prime}\right)=0 .
$$

For the remainder of this section, it will be assumed that kernels of operators are specified in a certain chosen orthonormal basis $\mathcal{P}^{\prime}$ of the same cardinality as $\mathscr{P}$,

$$
\begin{gathered}
\mathcal{P}^{\prime}=\left\{\phi_{\alpha} ; \alpha \in S\right\} \cup\left\{\phi_{\alpha}^{\prime} ; \alpha \in S\right\} \\
\left(\phi_{\alpha}^{\prime}, \phi_{\beta}^{\prime}\right)=\left(\phi_{\alpha}, \phi_{\beta}\right)=\delta(\alpha-\beta) \\
\left(\phi_{\alpha}, \phi_{\beta}^{\prime}\right)=0 .
\end{gathered}
$$

Let us define a linear operator $G_{0}$ by

$$
G_{0} \phi_{\alpha}=\phi_{\alpha}^{\prime} \text { and } G_{0} \phi_{\alpha}^{\prime}=-\phi_{\alpha} .
$$

Since $\mathscr{P}^{\prime}$ is an orthonormal basis, $G_{0}$ is the operator for which

$$
\left(\phi_{\alpha}, G_{0} \phi_{\beta}\right)=\left(\phi_{\alpha}^{\prime}, G_{0} \phi_{\beta}^{\prime}\right)=0
$$

and

$$
-\left(\phi_{\alpha}, G_{0} \phi_{\beta}^{\prime}\right)=\left(\phi_{\alpha}^{\prime}, G_{0} \phi_{\beta}\right)=\delta(\alpha-\beta) .
$$

One can define a linear transformation $C$ by

$$
C \phi_{\alpha}=\psi_{\alpha} \text { and } C \phi_{\alpha}^{\prime}=\psi_{\alpha}^{\prime} .
$$

Then

$$
C^{T} G C=G_{0} \quad\left(\text { where } C^{T} \text { is the transpose of } C\right)
$$


and

$-C^{T} G J C=D^{(2)}=\left[\begin{array}{ll}D & 0 \\ 0 & D\end{array}\right], \quad$ with $D$ positive definite diagonal in the basis $\mathscr{P}^{\prime}$

From (5.2),

$$
\mathcal{C}^{T} \bar{G}=\bar{G}_{0} C^{-i}
$$

Substituting this in (5.3),

$$
-G_{0} C^{-1} J C=D^{(2)},
$$

implying

$$
C^{-1}=J C=G_{0} D^{(2)}=\left[\begin{array}{cc}
0 & D \\
-D & 0
\end{array}\right] .
$$

Then

$$
\begin{aligned}
J^{2}=-I & \Rightarrow D^{2}=I \\
& \Rightarrow D=I \quad \text { and } D^{(2)}=I^{(2)} .
\end{aligned}
$$

Now (5.3) implies

$$
-G J=C^{-1} C^{-1} \text {. }
$$

Since $G J$ is bounded, $C^{-1}$ is bounded.

By (5.4),

$$
C=G C^{-1} G_{0}
$$

which must also be bounded.

Define $\mathcal{K}_{c}^{\prime}$ to be $\mathcal{K}$ with complex structure $G_{0}=C^{-1} J C$ and with inner product

$$
\langle\cdot, \cdot\rangle^{\prime}=\frac{1}{2}(\cdot, \cdot)-\frac{1}{2}\left(\cdot, G_{0} \cdot\right) \text {. }
$$

Let

$$
\hat{A}^{\prime}=C^{-1} \hat{A} C \text {. }
$$

Then $\hat{A}_{c}^{\prime}$ will denote $\hat{A}^{\prime}$ as an operator on complex space $\mathcal{H}_{c}^{\prime}$.

LemMA 5.6. $\operatorname{dom} \hat{A}^{\prime}=\operatorname{dom} \hat{A}_{c}^{\prime}$.

This follows from $\langle\psi, \psi\rangle^{\prime}=(\psi, \psi)$.

Lemma 5.7. $\psi \in \operatorname{dom} \hat{A}_{c} \Leftrightarrow C^{-1} \psi \in \operatorname{dom} \hat{A}_{c}^{\prime}$. 
This follows from

$$
\begin{aligned}
\left\langle\hat{A}_{c} \psi, \hat{A}_{c} \psi\right\rangle<\infty & \Leftrightarrow-(\hat{A} \psi, G J \hat{A} \psi)<\infty \\
& \Leftrightarrow\left(\hat{A} \psi, C^{-1} C^{-1} \hat{A} \psi\right)<\infty \\
& \Leftrightarrow\left(C^{-1} \hat{A} C C^{-1} \psi, C^{-1} \hat{A} C C^{-1} \psi\right)<\infty
\end{aligned}
$$

LEMMA 5.8. $\hat{A}_{c}^{\prime}$ is skew adjoint $\Leftrightarrow \hat{A}_{c}$ is skew adjoint.

Proof. First note that

$$
\begin{aligned}
& \langle\phi, \hat{A} \psi\rangle=\left(\phi, C^{-1} C^{-1} \hat{A} \psi\right)-i(\phi, G \hat{A} \psi) \\
& =\left\langle C^{-1} \phi, \hat{A}^{\prime} C^{-1} \psi\right\rangle^{\prime} \quad\left(\text { since } G=C^{-1} G_{0} C^{-1}\right) .
\end{aligned}
$$

Then, using Lemma 5.7, it follows that the skew adjointness of $\hat{A}_{c}$ is equivalent to the skew adjointness of $\hat{A}_{c}^{\prime}$.

LEMMA 5.9. $\hat{A}_{c}^{\prime}$ is skew adjoint if and only if $\hat{A}^{\prime}$ is skew adjoint and $G_{0} \hat{A}^{\prime}$ is self adjoint.

Proof $(\Rightarrow)$ If $\hat{A}_{c}^{\prime}$ is skew adjoint, it can be seen, after taking real and imaginary parts of $\left\langle\phi, \hat{A}^{\prime} \psi\right\rangle^{\prime}$, that $\hat{A}^{\prime}$ is skew Hermitian and $G_{0} \hat{A}^{\prime}$ is Hermitian on $\mathcal{H}$. To prove that $\hat{A}^{\prime}$ is skew adjoint on $\mathscr{K}$, we now need only to prove that $\operatorname{dom}\left(\hat{A}^{\prime}\right)^{T} \subseteq$ dom $\hat{A}^{\prime}$.

Suppose $\phi \in \operatorname{dom}\left(\hat{A}^{\prime}\right)^{T}$. According to the Frechet-Riesz theorem, this is equivalent to $\psi \rightarrow\left(\phi, \hat{A}^{\prime} \psi\right)$ being continuous.

This implies that

$$
\begin{aligned}
\psi \rightarrow\left(\phi, G_{0} \hat{A}^{\prime} \psi\right) & \text { is continuous, since } G_{0} \text { is bounded, } \\
\Rightarrow \psi & \rightarrow\left(\phi, \hat{A}^{\prime} \psi\right)-i\left(\phi, G_{0} \hat{A}^{\prime} \psi\right) \text { is continuous, } \\
& \Rightarrow \phi \in \operatorname{dom}\left(\hat{A}_{c}^{\prime}\right)^{\dagger}
\end{aligned}
$$

However, $\operatorname{dom}\left(\hat{A}_{c}^{\prime}\right)^{\dagger}=\operatorname{dom} \hat{A}_{c}^{\prime}$, since $\hat{A}_{c}^{\prime}$ is skew adjoint, by assumption and $\operatorname{dom} \hat{A}_{c}^{\prime}=\operatorname{dom} \hat{A}^{\prime}$, by Lemma 5.6. Therefore, $\operatorname{dom}\left(\hat{A}^{\prime}\right)^{T} \subseteq \operatorname{dom} \hat{A}^{\prime}$, as required, and so $\hat{A}^{\prime}$ must be skew adjoint.

Now to prove that $G_{0} \hat{A}^{\prime}$ is self adjoint, we shall use the following lemma:

Lemma 5.10. Assume that $\hat{A}_{c}^{\prime}$ is skew adjoint. Then

$$
\psi \in \operatorname{dom} \hat{A}^{\prime} \Rightarrow G_{0} \psi \in \operatorname{dom} \hat{A}^{\prime}
$$

and

$$
\hat{A}^{\prime} G_{0} \psi=G_{0} \hat{A}^{\prime} \psi
$$


Proof. Assume that $\hat{A}_{c}^{\prime}$ is skew adjoint.

This implies, by taking real and imaginary parts of the inner product in $\mathcal{K}_{c}$, that for all $\phi \in \operatorname{dom} \hat{A}^{\prime}\left(=\operatorname{dom} \hat{A}_{c}^{\prime}\right.$, by Lemma 5.6),

$$
\left(\psi, \hat{A}^{\prime} \phi\right)=-\left(\hat{A}^{\prime} \psi, \phi\right)
$$

and

$$
\left(\psi, G_{0} \hat{A}^{\prime} \phi\right)=-\left(\hat{A}^{\prime} \psi, G_{0} \phi\right) .
$$

Since $G_{0}$ is a skew symmetric compiex structure and is therefore orthogonal on $\mathcal{K}$, (5.11) may be replaced by

$$
\forall \phi \in \operatorname{dom} \hat{A}^{\prime}, \quad\left(G_{0} \psi, G_{0} \hat{A}^{\prime} \phi\right)=-\left(G_{0} \hat{A}^{\prime} \psi, G_{0} \phi\right)
$$

and

$$
-\left(G_{0} \psi, \hat{A}^{\prime} \phi\right)=\left(G_{0} \hat{A}^{\prime} \psi, \phi\right) .
$$

Since $\hat{A}_{c}^{\prime}$ is skew adjoint, this implies that

$$
G_{0} \psi \in \operatorname{dom} \hat{A}_{c}^{\prime}\left(=\operatorname{dom} \hat{A}^{\prime}\right)
$$

and

$$
-\hat{A}^{\prime} G_{0} \psi=-G_{0} \hat{A}^{\prime} \psi
$$

Now we shall use Lemma 5.10 to prove that

$$
\operatorname{dom}\left(G_{0} \hat{A}^{\prime}\right)^{T} \subseteq \operatorname{dom}\left(G_{0} \hat{A}^{\prime}\right),
$$

from which it follows that the Hermitian operator $G_{0} \hat{A}^{\prime}$ is self adjoint.

$$
\forall \psi \in \operatorname{dom} G_{0} \hat{A}^{\prime}, \quad\left(\phi, G_{0} \hat{A}^{\prime} \psi\right)=-\left(G_{0} \phi, \hat{A}^{\prime} \psi\right) .
$$

Suppose

$$
\phi \in \operatorname{dom}\left(G_{0} \hat{A}^{\prime}\right)^{T}
$$

That is,

$$
\begin{aligned}
& \begin{array}{l}
\exists \phi^{\prime}, \forall \psi \in \operatorname{dom}\left(G_{0} \hat{A}^{\prime}\right), \quad\left(\phi, G_{0} \hat{A}^{\prime} \psi\right)=\left(\phi^{\prime}, \psi\right) \\
\Rightarrow \exists \phi^{\prime}, \forall \psi \in \operatorname{dom}\left(G_{0} \hat{A}^{\prime}\right), \quad\left(-G_{0} \phi, \hat{A}^{\prime} \psi\right)=\left(\phi^{\prime}, \psi\right) \\
\Rightarrow \exists \phi^{\prime}, \forall \psi \in \operatorname{dom} \hat{A}^{\prime}, \quad\left(-G_{0} \phi, \hat{A}^{\prime} \psi\right)=\left(\phi^{\prime}, \psi\right) \\
\Rightarrow G_{o} \psi \in \operatorname{dom} \hat{A}^{\prime} \quad \text { and } \quad \phi^{\prime}=-\hat{A}^{\prime}\left(-G_{0} \phi\right) \quad\left(\text { since dom } G_{0} \hat{A}^{\prime} \supseteq \operatorname{dom} \hat{A}^{\prime}\right) \\
\text { (since we have already proven that } \hat{A}^{\prime} \text { is skew adjoint) }
\end{array} \\
& \Rightarrow \phi\left(=-G_{0} G_{0} \phi\right) \in \operatorname{dom} \hat{A}^{\prime} \quad \text { (by Lemma 5.10) }
\end{aligned}
$$


and

$$
\phi^{\prime}=\hat{A}^{\prime} G_{0} \phi=G_{0} \hat{A}^{\prime} \phi \quad \text { (by Lemma 5.10). }
$$

Therefore, $\operatorname{dom}\left(G_{0} \hat{A}^{\prime}\right)^{T}=\operatorname{dom}\left(G_{0} \hat{A}^{\prime}\right)$ and $G_{0} \hat{A}^{\prime}$ is Hermitian, and therefore is self adjoint.

We have now proved Lemma $5.9(\Rightarrow)$. The converse is obvious.

THEOREM 5.12. Suppose that there exists a complex structure J for which a given one parameter symplectic group $C(t)$ is a continuous one parameter unitary group on $\mathcal{K}_{c}=(\mathcal{K}, J,\langle\cdot, \cdot\rangle)$. Then $C(t)=\exp [\hat{A} t]$, with $\hat{A}$ similar to a skew adjoint operator on $\mathcal{K}$ and $G \hat{A}$ a self adjoint operator on $\mathcal{H}$.

Proof. By Stone's theorem, $C(t)=\exp \left[\hat{A}_{c} t\right]$, with $A_{c}$ skew adjoint on $\mathcal{K}_{c}$. Let $\hat{A}$ denote $\hat{A}_{c}$, considered as an operator on $\mathcal{H}$.

Consider $\hat{A}^{\prime}=C^{-1} \hat{A} C$, with $C$ as in (5.5). By Lemmas 5.8 and $5.9, \hat{A}^{\prime}$ is skew adjoint on $\mathcal{K}$ and $G_{0} \hat{A}^{\prime}$ is self adjoint on $\mathscr{K}$. Now

$$
\begin{aligned}
G \hat{A} & =G C \hat{A}^{\prime} C^{-1} \\
& =C^{-1} G_{0}^{T} \hat{A}^{\prime} C^{-1} \quad(\text { by }(5.2))
\end{aligned}
$$

Since $C^{-1}$ is bounded, $G \hat{A}$ is seen to be self adjoint. Theorem (5.12) establishes that unitarizable linear symplectic dynamics must be a Hamiltonian flow $e^{-G \hat{H} t}$, generated by the phase function $H=\frac{1}{2}(\mathbf{z}, \hat{H} \mathbf{z})$, with $\hat{H}$ a real self adjoint operator and that $G \hat{H}$ must be similar to a skew adjoint operator. This highlights the necessary stability of the classical dynamics. Recently, the Segal quantization procedure has been extended to some time-dependent linear dynamical systems and to some non-linear systems. Existence theorems in these cases have been proven only after imposing some conditions which ensure stability of the classical dynamics [23]. It seems that stability is a necessary condition for the existence of a canonical Hermitian structure on the real solution manifolds of these more complicated dynamical systems.

\section{Acknowledgements}

The author is grateful for the continued interest shown by Professor C. A. Hurst and for some technical advice of Dr. D. O’Brien. 


\section{References}

[1] A. Ashtekar and A. Magnon, "Quantum fields in curved space-time", Proc. Roy. Soc. London Ser. A 346 (1975), 375-94.

[2] J. Bognar, Indefinite inner product spaces (Springer, Berlin, 1974).

[3] P. Broadbridge, "Problems in the quantization of quadratic Hamiltonians", Hadronic J. 4 (1981), 899-948.

[4] P. Broadbridge and C. A. Hurst, "Existence of a complex stucture for quadratic Hamiltonians?" Ann. Physics 131 (1981), 104-117.

$[5 j \bar{Y}$. Broadibridge and C. A. Hurst, "Fermi-Dirac quantization of linear systems", Ann. Physics 137 (1981), 86-103.

[6] P. Broadbridge and C. A. Hurst, "Canonical forms for quadratic Hamiltonians", Phys. A 108 (1981), 39-62.

[7] J. M. Cook, “The mathematics of second quantization", Trans. Amer. Math. Soc. 74 (1953), 222-245.

[8] P.A.M. Dirac, The principles of quantum mechanics (Oxford University Press, London, 4th edition, 1958).

[9] S. A. Fulling, "Varieties of instability of a boson field in an external potential", Phys. Rev. D14 (1976), 1939-1944.

[10] F. Gallone and A. Sparzani, "Segal quantization", J. Math. Phys. 20 (1979), 1375-1384.

[11] F. Gallone and A. Sparzani, "On the uniqueness of the Segal quantization of linear Bose systems", J. Phys. A 14 (1981), 1341-1350.

[12] L. Gårding and A. S. Wightman, "Representations of the anticommutation relations", Proc. Nat. Acad. Sci. U.S.A. 40 (1954), 617-621.

[13] B. N. Harvey, "A spectral theorem for J. nonnegative operators", Trans. Amer. Math. Soc. 257 (1980), 387-396.

[14] B. S. Kay, "A uniqueness result in the Segal-Weinless approach to linear Bose fields", J. Math. Phys. 20 (1979), 1712-1714.

[15] M. G. Krein and Ju. L. Smul'jan, " $J$-polar representation of plus-operators", Mat. Issled. I (1966), 172-210; English transl., Amer. Math. Soc. Transl. (2) 85 (1969), 115-143.

[16] K. Maurin, General eigenfunction expansions and unitary representations of topological groups (Polish Scientific Publishers, Warsaw, 1968).

[17] M. A. Naimark, "An analogue of Stone's theorem in a space with an indefinite metric", Soviet Math. Dokl. 7 (1966), 1366-1368.

[18] S. M. Paneitz, "Unitarization of symplectics and stability for causal differential equations in Hilbert space", J. Funct. Anal. 41 (1981), 315-326.

[19] M. Reed and B. Simon, Methods of modern mathematical physics II. (Academic Press, New York, 1975).

[20] I. E. Segal, "Foundations of the theory of dynamical systems of infinitely many degrees of freedom I", Mat.-Fys. Medd. Danske Vid. Selsk. 31 (1959), No. 12.

[21] I. E. Segal, "Mathematical characterization of the physical vacuum for a linear Bose-Einstein field", Illinois J. Math. 6 (1962), 500-523.

[22] I. E. Segal, Mathematical problems of relativistic physics (Amer. Math. Soc., Providence, R. I., 1963).

[23] I. E. Segal, "Stability theory and quantization", in Differential geometric methods in mathematical physics, Lecture Notes in Physics 836 (Springer, Berlin, 1981).

[24] D. Shale and W. F. Stinespring, "States of the Clifford algebra", Ann. of Math. (2) 80 (1964), $365-381$.

[25] M. Weinless, "Existence and uniqueness of the vacuum for linear quantized fields", J. Funct. Anal. 4 (1969), 350-379.

[26] E. T. Whittaker, Analytical dynamics of particles and rigid bodies, Section 192 (Cambridge University Press, London, 4th edition, 1959). 